UNIVERSITY OF NOTTINGHAM

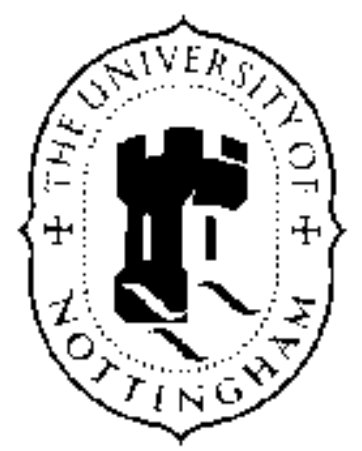

Discussion Papers in Economics

Discussion Paper

No. $06 / 08$

\title{
EXISTENCE, UNIQUENESS AND SOME COMPARATIVE STATICS FOR RATIO- AND LINDAHL EQUILIBRIA: NEW WINE IN OLD BOTTLES
}

by Wolfgang Buchholz, Richard Cornes and Wolfgang Peters 


\title{
Existence, Uniqueness and Some Comparative Statics for Ratio- and Lindahl Equilibria: New Wine in Old Bottles
}

by

\author{
Wolfgang Buchholz, Richard Cornes and Wolfgang Peters
}

September 04, 2006

\begin{abstract}
We present a rigorous, yet elementary, demonstration of the existence of a unique Lindahl equilibrium under the assumptions that characterize the standard $n$-player public good model. Indeed, our approach, which exploits the aggregative structure of the public good model, lends itself to a transparent geometric representation. Moreover, it can handle the more general concept of the cost-share or ratio equilibrium. Finally, we indicate how it may be exploited to facilitate comparative static analysis of Lindahl and cost share equilibria.
\end{abstract}

Keywords: Public goods, Lindahl equilibrium, ratio equilibrium.

JEL classifications: H41.

Wolfgang Buchholz, Department of Economics, University of Regensburg, 93040 Regensburg, Germany (wolfgang.buchholz@wiwi.uni-regensburg.de); Richard Cornes, School of Economics, University of Nottingham, Nottingham NG 2RD, UK (rccornes@aol.com); Wolfgang Peters, Department of Economics, European University Viadrina, PO Box 1786, 15207 Frankfurt (Oder), Germany (Peters@euv-frankfurt-o.de) 


\section{Introduction}

The Lindahl equilibrium represents the traditional solution concept through which a cooperative outcome in a public-good economy is specified. The description and analysis of Lindahl equilibria thus has been a standard topic in the theory of public goods for a very long time. It is therefore surprising that no simple existence proof for a Lindahl equilibrium seems to be available, even in the textbook case when a single public good is produced by a constant returns to scale technology and there is an arbitrary number of agents. The diagrams that are normally used to visualize the existence of a Lindahl solution only apply to the case with two agents and cannot be generalized to the case of more agents (see ,e.g., Atkinson and Stiglitz, 1980, pp. 509-510, Myles, 1995, pp. 272-275, Cornes and Sandler, 1996, pp. 201-205, Thomson, 1999, or Hindriks and Myles, 2006, pp. 113-117). The more rigorous and general existence proofs, however, are - by, e g., making use of Kakutani's fixed point theorem - mathematically rather demanding (see ,e.g., Foley, 1967, Milleron, 1972, Roberts, 1974, Kaneko, 1977, or Mas-Colell and Silvestre, 1989) and quite different from the standard treatment of public good theory. Moreover, the problem of uniqueness which is treated extensively for other types of economic equilibria has not attracted much attention in the context of the Lindahl solution until now.

In order to close this gap we describe in the present paper how the existence and the uniqueness issues can be dealt with by an elementary method that, on the one hand, is related to the analysis of aggregative games as developed by Cornes and Hartley (2007) and, on the other hand, is inspired by the graphical devices already presented in the seminal papers by Bowen (1943) and Samuelson (1955). Exploiting this approach it is also possible to include proportional cost-share or ratio equilibria (see Kaneko, 1977, or Silvestre, 2003, for a comprehensive discussion) - a natural generalization of Lindahl equilibria for the case of nonconstant marginal costs for producing the public good - from the outset without incurring any essential complication. After presenting the general theoretical framework in Section 2 and discussing the existence and uniqueness issue in Section 3, we show in Section 4 how this method expedites comparative static analysis of Lindahl and ratio equilibria. Section 5 concludes with some remarks on how Bowen's (1943) and Samuelson's (1955) venerable contributions can be interpreted from the viewpoint of the present paper. 


\section{The Framework}

In an economy consisting of $n$ agents, $x_{i}$ denotes agent $i$ 's private consumption and $G$ is public good supply. Agent $i$ is endowed with an amount $y_{i}$ of the private good (his "income") which can be transformed into units of the public good. The aggregate budget constraint is

$$
\sum_{i=1}^{n} x_{i}+C(G)=Y
$$

where $C(G)$ is the total cost of producing $G$ and $Y=\sum_{i=1}^{n} y_{i}$ is the total income of all agents. The cost function is assumed to have $C(0)=0$, to be twice differentiable for $G>0$ and to be left-hand differentiable at $G=0$ with $C^{\prime}(0)>0$ as its derivative. For the first and second derivatives we assume $C^{\prime}(G)>0$ for all $G>0$ and $C^{\prime \prime}(G) \geq 0$ for all $G \geq 0$, so that we have non-decreasing marginal costs.

The preferences of agent $i$ are given by the utility function $u_{i}\left(x_{i}, G\right)$ that is defined for all $x_{i} \geq 0$ and all $G \geq 0$. Each utility function is assumed to be continuous on its domain and to be twice continuously differentiable and strictly quasi-concave on $x_{i}>0$ and $G>0$. Furthermore, we suppose throughout the whole analysis that the public good is at least weakly non-inferior for every agent $i$. By $G_{i}(p)$ we now denote agent $i$ 's Marshallian pseudo-demand function for the public good when agent $i$ is endowed with income $y_{i}$ and has to bear the individual cost share $p_{i}$ in the total cost of the public good. Given any $p_{i}$, the value of $G_{i}\left(p_{i}\right)$ is obtained from maximizing utility $u_{i}\left(y_{i}-p_{i} C(G), G\right)$ for all $G>0$, where for the sake of convenience we allow for $p_{i}>1$ such that at the outset $p_{i}$ need not be a true cost share. If the cost function $C(G)$ is linear, $p_{i}$ corresponds to the personalized public-good price as in the standard Lindahl model, and in this case $G_{i}\left(p_{i}\right)$ is the conventional Marshallian demand function of agent $i$.

Define $\bar{p}_{i}:=\sup \left\{p_{i}: G_{i}\left(p_{i}\right)>0\right\} \in(0, \infty)$ for each agent $i$. By weak non-inferiority of the public good and strict convexity of the indifference curves each demand function $G_{i}\left(p_{i}\right)$ then is strictly decreasing in the cost share $p_{i}$ on $\left(0, \bar{p}_{i}\right)$, which for the general case is shown in 
Appendix A1. For the constant marginal cost case, this is a standard result in basic microeconomics. Furthermore, $\lim _{p_{i} \rightarrow \bar{p}_{i}} G_{i}\left(p_{i}\right)=0$ and $\lim _{p_{i} \rightarrow 0} G_{i}\left(p_{i}\right)=\infty$. (The first lim-property is obvious from continuity, the second follows by an indirect proof: Assume that $G_{i}\left(p_{i}\right)$ were bounded from above by some finite $\bar{G}_{i}$. Then consider the indifference curve passing through $\left(y_{i}, \bar{G}_{i}\right)$. By our assumptions, this has a finite non-zero slope there. Choose some $\tilde{G}>\bar{G}_{i}$ and observe that $\lim _{p_{i} \rightarrow 0}\left(y_{i}-p_{i} C(\tilde{G})\right)=y_{i}$. Consequently, for some sufficiently low cost-share parameter $\tilde{p}_{i}$, agent $i$ 's possibility curve defined by $\left(y_{i}-\tilde{p}_{i} C(G), G\right)$ intersects the indifference curve through $\left(y_{i}, \bar{G}_{i}\right)$. Given $\tilde{p}_{i}$ public-good demand of agent $i$ then would be higher than $\bar{G}_{i}$ which is a contradiction.)

These considerations imply that the inverse function of $G_{i}\left(p_{i}\right)$, denoted by $p_{i}(G)$, is defined for all $G>0$. It is strictly decreasing in $G$, never becomes zero and takes on any value in $\left(0, \bar{p}_{i}\right)$

\section{Existence and Uniqueness of Proportional Cost-Share Equilibria}

In the present public good economy a Lindahlian cost-share (or ratio) equilibrium is defined by an $n$-tuple of non-negative personalized cost shares $\left(p_{1}^{L}, \ldots, p_{n}^{L}\right)$, an $n$-tuple of individual consumption levels $\left(x_{1}^{L}, \ldots, x_{n}^{L}\right)$ and a supply level of the public good $G^{L}>0$ such that

(i) $\quad G^{L}$ maximizes utility $u_{i}\left(y_{i}-p_{i}^{L} C(G), G\right)$ of agent $i$ among all $G$ if agent $i$ is confronted with the personalized public good price $p_{i}^{L}$,

(ii) $\quad x_{i}^{L}=y_{i}-p_{i}^{L} C\left(G^{L}\right)$ for all $i=1, \ldots, n$, which is non-negative given (i),

(iii) $\quad \sum_{i=1}^{n} p_{i}^{L}=1$, which means that the allocation $\left(x_{1}^{L}, \ldots, x_{n}^{L}, G^{L}\right)$ is feasible according to (1).

It directly follows from condition (iii), that in a ratio equilibrium $p_{i}^{L} \leq 1$ must hold for each agent $i$ such that true cost shares are obtained. With the help of the inverse pseudo- 
demand functions $p_{i}(G)$ existence and uniqueness of such a proportional cost-share equilibrium can now be established in a very simple way using the public good level $G$ as a parameter. This procedure thus parallels the "aggregative games approach" by Cornes and Hartley (2007), which in the context of public goods has until now only been used to describe noncooperative Cournot-Nash equilibria. In order to make this method also applicable for the analysis of cooperative Lindahl equilibria we introduce a new function:

$$
P(G):=\sum_{i=1}^{n} p_{i}(G)
$$

This aggregate price function $P(G)$ corresponds to total willingness to pay for the public good when public-good supply is $G$ and, in addition, all agents demand the amount $G$ as cost-share takers. For the case of quasi-linear utility function such an addition of marginal willingness to pay has already been an element of Bowen's analysis (1943) but was - as Samuelson's (1955) graphical device - not used to characterize Lindahl equilibria until now. The function $P(G)$ is defined for all $G>0$ and takes on all values in $(0, \bar{P})$ where $\bar{P}:=\sum_{i=1}^{n} \bar{p}_{i}$. It follows from strict monotonicity and continuity of all functions $p_{i}(G)$ that $P(G)$ is strictly decreasing and continuous on $(0, \infty)$. The following result therefore holds:

Proposition 1: If $\bar{P}>1$ there exists a unique Lindahlian cost-share equilibrium with $G^{L}>0$ and $p_{i}^{L}>0$ for all $i=1, \ldots, n$.

Proof: Given our assumptions we have $P(G)>1$ for small values of $G$ but $P(G)<1$ for high values of $G$. The mean value theorem then implies that there exists some $\hat{G}>0$ such that $P(\hat{G})=1$ as depicted in Figure 1 . 


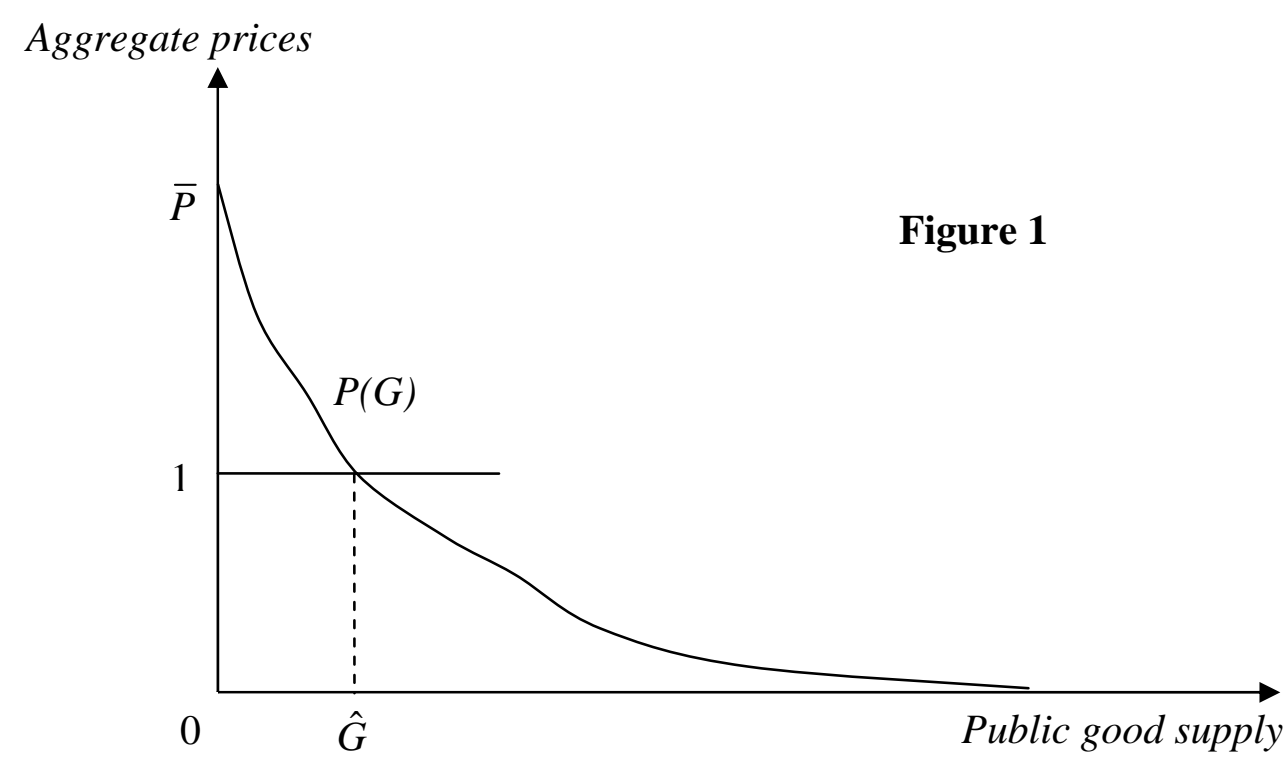

Let individual cost-shares now be defined by $\hat{p}_{i}:=p_{i}(\hat{G})>0$ for each agent $i$. Then conditions (i) and (iii) are obviously fulfilled given $\left(\hat{p}_{1}, \ldots, \hat{p}_{n}\right)$ and $\hat{G}$. If private consumption is $\hat{x}_{i}:=y_{i}-\hat{p}_{i} C(\hat{G})$ for each agent $i=1, \ldots, n$, condition (ii) holds by definition. Therefore, $\left(\hat{X}_{1}, \ldots, \hat{X}_{n}, \hat{G}\right)$ is a ratio equilibrium with personalized cost shares $\left(\hat{p}_{1}, \ldots, \hat{p}_{n}\right)$. This proves existence. For the uniqueness part let some cost-share equilibrium with public-good supply $G^{L}$ be given. Then, condition (iii) implies $P\left(G^{L}\right)=1=P(\hat{G})$ since from condition (i) we have $p_{i}\left(G^{L}\right)=p_{i}^{L}$ for each agent $i$. Because $P(G)$ is strictly decreasing in $G$ this implies $G^{L}=\hat{G}$. We then obtain $p_{i}^{L}=p_{i}\left(G^{L}\right)=p_{i}(\hat{G})=\hat{p}_{i}$ and $\hat{x}_{i}=y_{i}-\hat{p}_{i} \hat{G}=y_{i}-p_{i}^{L} G^{L}=x_{i}^{L}$ for all agents $i=1, \ldots, n$ which shows that any ratio equilibrium must coincide with the alloca$\operatorname{tion}\left(\hat{x}_{1}, \ldots, \hat{x}_{n}, \hat{G}\right)$.

QED.

In the standard textbook case with indifference curves that are tangential to the $G$-axis $\bar{P}=\infty$ is obtained such that, according to Proposition 1, existence and uniqueness of a Lindahl equilibrium is ensured for any cost function. Note that the construction underlying the proof of Proposition 1 ensures that a cost-share equilibrium is Pareto-optimal since the conditions that characterize ratio equilibria coincide with the Samuelson condition for optimality. 


\section{Some Comparative Statics}

We now analyze the effects on public-good supply and individual welfare when the composition of the agents and thus the aggregate price function $P(G)$ is changed and the cost function $C(G)$ is given. In particular we consider the case in which one of the original individuals, say agent $k$, is substituted by another type of agent with either higher income $\tilde{y}_{k}$ or a utility function $\tilde{u}_{k}\left(x_{k}, G\right)$ that exhibits a stronger preference for the public good. Precisely, the change of preferences is described by the assumption that agent $k$ 's marginal willingness to pay for the public good, i.e. his marginal rate of substitution $\pi_{k}\left(x_{k}, G\right)$ between the public of the private good, is for any $\left(x_{k}, G\right)$ higher under $\tilde{u}_{k}$ than under the original utility function $u_{k}$. In both cases the value of the new pseudo-demand for the public good is increased everywhere, i.e. $\tilde{G}_{k}\left(p_{k}\right)>G_{k}\left(p_{k}\right)$ is obtained for any cost-share level $p_{k}>0$ when we assume strong normality of the public good in the case of an income increase. Such a change of demand curves is obvious if the underlying technology exhibits constant returns to scale. For the general case of a convex cost function this result is shown in Appendix A2. Then for the inverse demand function we have $\tilde{p}_{k}(G)>p_{k}(G)$ for every $G>0$. Consequently, $\tilde{P}(G):=\tilde{p}_{k}(G)+\sum_{i \neq k} p_{i}(G)>\sum_{i=1}^{n} p_{i}(G)=P(G)$.

In the other scenario, an additional agent $n+1$ with income $y_{n+1}$ and the utility function $u_{n+1}\left(x_{i}, G\right)$ joins the economy whereas the original agents $i=1, \ldots, n$ remain the same. If $p_{n+1}(G)$ then denotes this agent's inverse pseudo-demand function for the public good we again get $\tilde{P}(G):=\sum_{i=1}^{n+1} p_{i}(G)>\sum_{i=1}^{n} p_{i}(G)=P(G)$ for any $G>0$. Then we directly obtain the following basic comparative statics result for cost-share equilibria. (For a more intricate analysis of the comparative statics of Lindahl equilibria see also Sertel, 1994, Sertel and Yilmiz, 1996, and Thomson, 1999.)

Proposition 2: Assume that one agent in the original economy gets a higher income or that he is substituted by another agent with a higher preference for the public good or that a new agent enters the economy. In the Lindahl equilibrium then public good supply and the utility levels of all unaltered agents are increased. 
Proof: Let $\tilde{G}^{L}$ be public-good supply in the (new) cost-share equilibrium after one of the changes considered in Proposition 2 has been made. If $\tilde{P}(G)$ denotes the after-change aggregate price function $\tilde{G}^{L}$ fulfils $\tilde{P}\left(\tilde{G}^{L}\right)=1$ by Proposition 1 . Since $\tilde{P}(G)>P(G)$ for all $G>0$ and $P(G)$ and $\tilde{P}(G)$ both are strictly decreasing $\tilde{G}^{L}>G^{L}$ is obtained. As the inverse pseudodemand functions $p_{i}(G)$ are strictly decreasing in $G$ the Lindahlian cost share $\tilde{p}_{i}^{L}$ of any agent $i$ who is unaffected by the change therefore becomes smaller, i.e. $\tilde{p}_{i}^{L}=p_{i}\left(\tilde{G}^{L}\right)<p_{i}\left(G^{L}\right)=p_{i}^{L}$. Confronted with the lower cost share $\tilde{p}_{i}^{L}$ each of these invariant agents will then clearly attain higher utility after the change.

QED.

Note that in the case where the agent with the changing income has quasi-linear preferences of the form $u_{k}\left(x_{k}, G\right)=x_{k}+v_{k}(G)$ the only change in the Lindahl solution is an increase of private consumption of agent $k$ which is equal to the income increase. Thus, the result of Proposition 2 requires the assumption of strong normality when an increase of income is considered.

\section{A Historical Remark}

A diagram that looks like our Figure 1 was already used by Bowen (1943, p. 31) to characterize the "ideal output" of a public good. In his paper, however, no reference to Lindahl (1919) was made. There was no clear distinction between the marginal and the average cost function, and instead of the inverse pseudo-demand curve $P(G)$ as in our equation (1), Bowen (1943, p. 30) constructed a "curve of total marginal substitution ... expressing the amount of money the members of the group would be willing to give up in order to obtain successive units of education (the public good, W.B, R.C. and W.P)." He only made some short remarks that this function should somehow be related to individual demand functions.

Samuelson (1955, pp. 353-354) took up Bowen's diagram in order to compare his own graphical exposition of public-good theory with earlier approaches. He referred to Lindahl in this context (without having "access to this important work", see Footnote 8 on p. 354 of Samuelson, 1954), and clarified that the use of the marginal cost function is required to determine a Pareto optimal solution. But he also remained quite opaque concerning the nature of the $P(G)$-curve, saying that it should come about by aggregating marginal rates of substitution along indifference curves of the different agents involved. According to Samuelson (1955, p. 353) "these schedules look like demand curves", not stating explicitly that he actu- 
ally meant Hicksian demand curves. By not using Marshallian pseudo-demand functions of cost-share as in Lindahl's two-agent-model, Samuelson missed Lindahl's true concerns and made things more complicated. Hicksian and Marshallian demand curves only coincide in special situations, e.g. if utility functions are quasi-linear of the form $u_{i}\left(x_{i}, G\right)=x_{i}+v_{k}(G)$. Only in this case it makes sense to refer to an addition of mrs-curves or demand curves without further qualification as it is often done in textbooks. (For some non-technical discussion of the Bowen-Samuelson graphical device see Musgrave, 1959, pp. 57-59, Danziger, 1976, and Batina and Ihori, 2005, pp. 13-14.)

In the end, Samuelson (1955, p. 354)) gave a rather negative assessment of both Bowen's and Lindahl's graphical devices stating that "there is something circular and unsatisfactory about both the Lindahl and the Bowen construction." In contrast, the purpose of this paper is to show that Bowen's idea if correctly interpreted can be very helpful to establish central features of the Lindahl model in a direct and rigorous way.

\section{Appendix}

\section{A1 The Effects of a Changing Cost Share on Public Good Demand}

Let the cost function $C(G)$ be given as in the main text and consider some agent $i$. We want to show that a higher cost share implies a lower public-good demand of agent $i$. For an indirect proof assume that we have $\tilde{p}_{i}>p_{i}$ but, simultaneously, $G_{i}\left(\tilde{p}_{i}\right) \geq G_{i}\left(p_{i}\right)$. For privategood demand before and after the cost share increase we then obtain $x_{i}\left(\tilde{p}_{i}\right)<x_{i}\left(p_{i}\right)$. Let $\pi_{i}\left(x_{i}, G\right)$ denote agent $i$ 's marginal rate of substitution between the public and the private good at any $\left(x_{i}, G\right)$ which by weak non-inferiority does not increase when public good supply grows and private consumption falls. Observing the marginal conditions resulting from agent i's utility maximization, we then get

$$
\tilde{p}_{i} C^{\prime}\left(G\left(\tilde{p}_{i}\right)\right)=\pi_{i}\left(x_{i}\left(\tilde{p}_{i}\right), G_{i}\left(\tilde{p}_{i}\right)\right) \leq \pi_{i}\left(x_{i}\left(p_{i}\right), G_{i}\left(p_{i}\right)\right)=p_{i} C^{\prime}\left(G\left(p_{i}\right)\right)
$$

This contradicts our assumptions as from $C^{\prime \prime}(G) \geq 0$ we have $C^{\prime}\left(G_{i}\left(\tilde{p}_{i}\right)\right) \geq C^{\prime}\left(G_{i}\left(p_{i}\right)\right)$. Therefore, each agent $i$ 's pseudo-demand function $G_{i}\left(p_{i}\right)$ for the public good is strictly decreasing in the cost-share parameter $p_{i}$. 


\section{A2 The Effects of Changing Income and Preferences on Public-Good Demand}

Let some cost share $p_{k}$ of agent $k$ be given. Assume, for an indirect proof, that we have $\tilde{G}_{k}\left(p_{k}\right) \leq G_{k}\left(p_{k}\right)$ either after an increase of agent $k$ 's income or by an intensification of his preferences for the public good as described in the main text. For agent $k$ 's private consumption before and after the change we then would obtain $\tilde{x}_{k}\left(p_{k}\right) \geq x_{k}\left(p_{k}\right)$ with a strong inequality in the case of an increasing income. Analogous to the analysis in Appendix A1 this would imply

$$
p_{k} C^{\prime}\left(\tilde{G}\left(p_{k}\right)\right)=\pi_{k}\left(\tilde{x}\left(p_{k}\right), \tilde{G}\left(p_{k}\right)\right)>\pi_{k}\left(x\left(p_{k}\right), G\left(p_{k}\right)\right)=p_{k} C^{\prime}\left(G\left(p_{k}\right)\right) .
$$

Here, the strong inequality follows from strong normality of the public good in the case of an income change and, in the case of a preference change, from the definition of stronger preferences for the public good and weak normality. This inequality, however, contradicts the assumed convexity of the cost function $C(G)$.

\section{References:}

Atkinson, A. B. and J. Stiglitz (1980), Lectures on Public Economics, McGraw-Hill, New York.

Batina, R. G. and T. Ihori (2005), Public Goods: Theory and Applications, Springer, Berlin and Heidelberg.

Bowen, H. (1943), The Interpretation of Voting in the Allocation of Economic Resources, Quarterly Journal of Economics 58, 27-48.

Cornes, R. C. and T. Sandler (1996), The Theory of Externalities, Public Goods and Club Goods, $2^{\text {nd }}$ Edition, Cambridge University Press. Cambridge, England.

Cornes, R. C. and R. Hartley (2007), Aggregative Public Good Games, Journal of Public Economic Theory 9, forthcoming. 
Danziger, L. (1976), A Graphical Representation of the Nash and Lindahl Equilibria in an Economy with a Public Good, Journal of Public Economics 6, 295-303.

Foley, D. (1967), Resource Allocation and the Public Sector, Yale Economic Essays 7, 45-98.

Hindriks, J., and G. D. Myles (2006), Intermediate Public Economics, MIT Press, Cambridge, MA.

Kaneko, M. (1977), The Ratio Equilibrium and a Voting Game in a Public Good Economy, Journal of Economic Theory 16, 123-136.

Mas-Colell, A. and J. Silvestre (1989), Cost Share Equilibria: A Lindahlian Approach, Journal of Economic Theory 47, 239-256.

Milleron, J.-C. (1972), Theory of Value with Public Goods, Journal of Economic Theory 5, 419-477.

Musgrave, R. A. (1959), The Theory of Public Finance, McGraw Hill, New York.

Myles. G. D. (1995), Public Economics, Cambridge, England, et al., Cambridge University Press.

Roberts, D. (1974), The Lindahl Solution for Economics with Public Goods, Journal of Public Economics 3, 23-42.

Samuelson, P. A. (1955), Diagrammatic Exposition of a Theory of Public Expenditure, Review of Economics and Statistics 37, 350-356.

Sertel, M. (1994), Manipulating Lindahl Equilibria via Endowments, Economic Letters 46, 167-171.

Sertel, M. and M. Yildiz (1998), The Lindahl Solution with Changing Population and Resources, Mathematical Social Sciences 35, 151-163. 
Silvestre, J. (2003), Wicksell, Lindahl and the Theory of Public Goods, Scandinavian Journal of Economics 105, 527-553.

Thomson, W. (1999), Economies with Public Goods: An Elementary Geometric Exposition, Journal of Public Economic Theory 1, 139-176. 